\title{
ELECTROWEAK MEASUREMENTS AT CDF *
}

\author{
A. $\operatorname{SIDOTI}^{\dagger}$ \\ Laboratoire de Physique Nuclèaire et de Hautes Energies \\ Universitè "Pierre et Marie Curie" (Paris VI) \\ 4. Place Jussieu \\ 75252 Paris Cedex 05, France
}

\begin{abstract}
We present some recent measurements on electroweak physics using data collected by the CDF experiment at the Tevatron proton anti-proton collider $(\sqrt{s}=$ $1.96 \mathrm{TeV}$ ) at Fermilab (Batavia, Ill, USA).
\end{abstract}

\section{Introduction}

The CDF electroweak physics program is one of the key components of the RunII. Electroweak measurements are complementary to those performed at $e^{+} e^{-}$machines (LEP and SLD). The former can produce a larger number of $\mathrm{W}$ bosons and can produce $Z / \gamma^{\star}$ at higher invariant mass with respect of the latters.

We will review CDF electroweak physics measurements using data collected from February 2002. The integrated luminosity of data ranges from $64 \mathrm{pb}^{-1}$ to $\sim 200 \mathrm{pb}^{-1}$ depending on the measurement.

\section{2. $\mathrm{W}$ and $\mathrm{Z}$ inclusive cross section measurements}

Due to the high branching ratios and clean signature $\mathrm{W}$ and $\mathrm{Z}$ bosons are identified through their leptonic decays. Inclusive cross sections of both $\mathrm{W}$ and $\mathrm{Z}$ have been measured using all the three leptons: electrons, muons and taus and using all the available subdetectors of CDF extending in particular the geometric acceptance at high pseudorapidity $\eta^{\mathrm{a}}$. All the measurements

* On behalf of the CDF collaboration

${ }^{\dagger}$ Work supported by Research Training Network of E.U. "Probe of New Physics" HPRNCT-2002-00292 Contract

${ }^{\mathrm{a}} \eta$ is related to the azimuthal angle through the relation $\eta=-\log \tan (\theta / 2)$ 
performed are shown in Fig.1 and are in agreement with the theoretical predictions ${ }^{1}$.
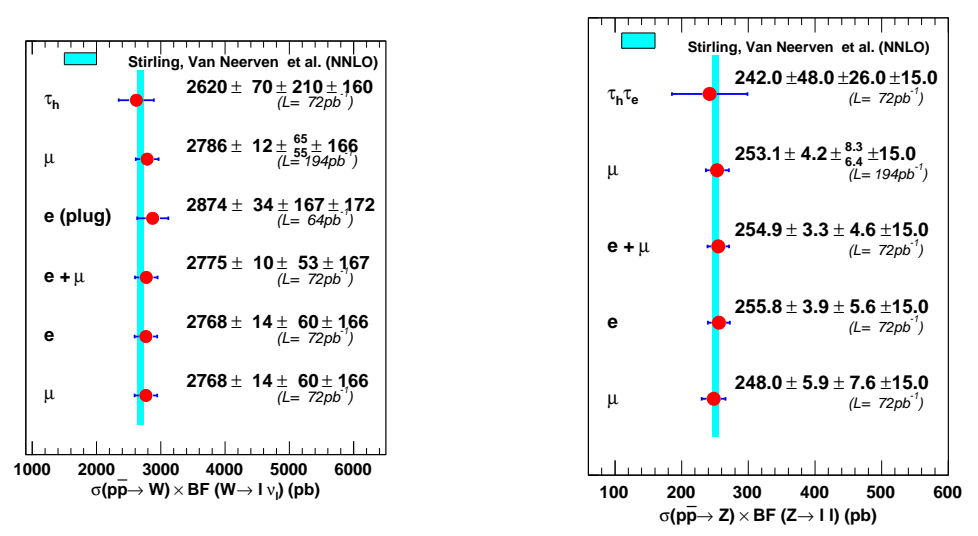

Figure 1. $\quad \sigma(p \bar{p} \rightarrow W) \times B F\left(W \rightarrow \ell \nu_{\ell}\right)($ left $)$ and $\sigma(p \bar{p} \rightarrow Z) \times B F(Z \rightarrow \ell \ell)$ (right) measured at CDF. The blue band indicates the theoretical (NNLO) predictions.

A stringent test of the Standard Model can be performed by evaluating the ratio $R$ of the $\mathrm{W}$ and $\mathrm{Z}$ boson cross sections:

$$
R=\frac{\sigma(p \bar{p} \rightarrow W) \times \mathrm{BF}\left(W \rightarrow \ell \nu_{\ell}\right)}{\sigma(p \bar{p} \rightarrow Z) \times \mathrm{BF}(Z \rightarrow \ell \ell)}
$$

that can be written as

$$
R=\frac{\sigma(p \bar{p} \rightarrow W)}{\sigma(p \bar{p} \rightarrow Z)} \times \frac{\Gamma(Z)}{\Gamma(Z \rightarrow \ell \ell)} \times \frac{\Gamma\left(W \rightarrow \nu \nu_{\ell}\right)}{\Gamma(W)} .
$$

Inserting the theoretical values of the total cross sections and of the partial width $W \rightarrow \ell \nu_{\ell}$ and the experimental partial and total widths of the $\mathrm{Z}$ boson from LEP, it is possibile to extract indirectly the total width of the $\mathrm{W}$ boson. The measured value by $\mathrm{CDF}$ is:

$$
\begin{aligned}
& \Gamma(W)=2079 \pm 41 \mathrm{MeV} \quad \mathrm{CDF} e+\mu \text { channel } \int \mathcal{L} d t=72 \mathrm{pb}^{-1} \\
& \Gamma(W)=2056 \pm 44 \mathrm{MeV} \quad \mathrm{CDF} \mu \text { channel } \int \mathcal{L} d t=194 \mathrm{pb}^{-1}
\end{aligned}
$$

well in agreement with both the PDG world average ${ }^{3}$ and the theoretical predictions:

$$
\Gamma(W)_{\mathrm{PDG}}=2118 \pm 41 \mathrm{MeV} \quad \Gamma(W)_{\mathrm{Th} .}=2092.1 \pm 2.5 \mathrm{MeV}
$$




\section{W Boson Asymmetries}

A precise charge asymmetry as a function of rapidity of the $\mathrm{W}$ boson provides constraints on the parton fluxes of the incoming protons and therefore provides a better determination of the parton distribution functions (pdf). The leptonic decay of the $\mathrm{W}$ boson makes difficult to measure directly the $\mathrm{W}$ rapidity itself. Instead, the electron asymmetry is measured that is the convolution of the $\mathrm{W}$ production charge asymmetry and the $\mathrm{V}$-A asymmetry from the $\mathrm{W}$ decay. The lepton charge aymmetry is defined as:

$$
A\left(\eta_{\ell}\right)=\frac{d \sigma_{+} / d \eta_{\ell}-d \sigma_{-} / d \eta_{\ell}}{d \sigma_{+} / d \eta_{\ell}+d \sigma_{-} / d \eta_{\ell}}
$$

where $\eta_{\ell}$ is the pseudorapidity of the lepton. CDF has measured the $\mathrm{W}$ charge asymmetry in the electron channel. The result as a function of the electron rapidity is shown in Fig.2. The measurement has been corrected for the effect of charge mis-identification and background contributions both dependent on the pseudorapidity. An additional selection $35<E_{T}<45$ $\mathrm{GeV}$ is applied on the electron energy to increase the sensitivity to different pdf. In fact the direction of an electron with higher energies is closer to the direction of emission of the $\mathrm{W}$ boson enhancing therefore the $\mathrm{W}$ production asymmetry $^{4}$

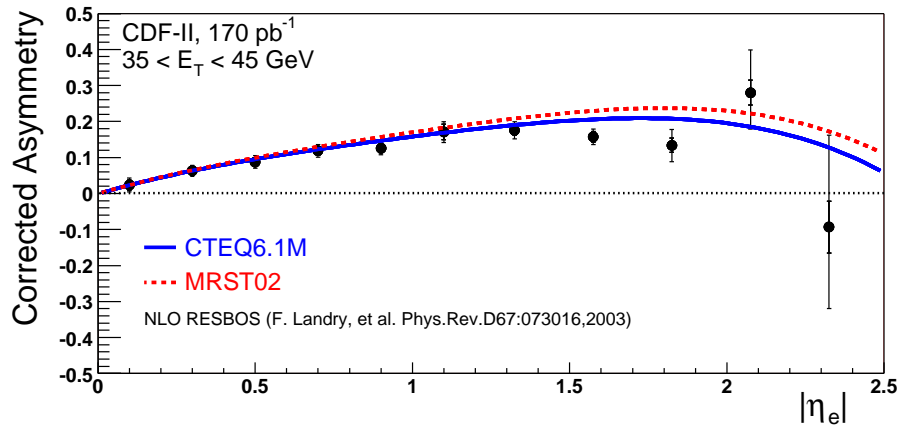

Figure 2. W charge asymmetry as a function of the electron pseudorapidity, with $35<E_{T}<45 \mathrm{GeV}$. Comparison with expectations from different pdf are shown.

\section{Di-Boson Production}

Di-boson processes probe electroweak gauge bosons interactions as well as sources of physics beyond Standard Model. 


\section{1. $W \gamma$ and $Z_{\gamma}$ production}

When one of the bosons is a photon the main backgounds are $\pi_{0}$ and jets faking a photon. To reduce these, CDF exploits the high spatial resolution of the electromagnetic calorimeter provided by the showermax detector ${ }^{5}$ and a track isolation criterium. The $W \gamma \rightarrow \ell \nu_{\ell} \gamma$ process has been studied by CDF in the electron and muon channel. Candidate events are selected requiring a high- $P_{T}$ momentum lepton with large missing transverse energy and $M_{T}(\ell, \nu)<120 \mathrm{GeV} / c^{2}$. The presence of a photon with $E_{T}>7 \mathrm{GeV}$ well separated from the lepton $\left(\Delta R(\ell, \gamma)>0.7^{\mathrm{b}}\right)$ is required. The cross section times branching fraction measured is:

$$
\sigma \times \mathrm{BF}\left(W \gamma \rightarrow \ell \nu_{\ell} \gamma\right)=18.1 \pm 1.6 \text { (stat.) } \pm 2.4 \text { (syst.) } \pm 1.2 \text { (lum.) } \mathrm{pb}^{-1}
$$

The predicted cross section including the photon acceptance is ${ }^{7}$ :

$$
\sigma \times \mathrm{BF}\left(W \gamma \rightarrow \ell \nu_{\ell} \gamma\right)_{\mathrm{Th} .}=19.3 \pm 1.4 \mathrm{pb}
$$

The photon transverse energy is shown in Fig.3.

$Z \gamma \rightarrow \ell \ell \gamma$ candidates are selected requiring two oppositely charged high momentum leptons with a dilepton invariant mass such that $M_{\ell, \ell}>40$ $\mathrm{GeV} / c^{2}$. The photon selection is the same of the $W \gamma \rightarrow \ell \nu_{\ell} \gamma$. The cross section times branching fraction measured is ${ }^{6}$ :

$$
\sigma \times \mathrm{BF}(Z \gamma \rightarrow \ell \ell \gamma)=4.6 \pm 0.5(\text { syst }+ \text { stat }) \pm 0.3(\text { lum. }) \mathrm{pb}^{-1}
$$

The predicted cross section including the photon acceptance is ${ }^{7}$ :

$$
\sigma \times \mathrm{BF}\left(W \gamma \rightarrow \ell \nu_{\ell} \gamma\right)_{\mathrm{Th} .}=4.5 \pm 0.3
$$

\subsection{WW Production}

WW production has been studied at CDF in the $W W \rightarrow \ell \nu_{\ell} \ell^{\prime} \nu_{\ell}^{\prime}$ channel looking for two oppositely charged high $P_{T}$ leptons and large missing transverse energy in the final state. Using and integrated luminosity of 184 $\mathrm{pb}^{-1} 17$ candidate events have been found with an estimated background of $5.0_{-0.8}^{+2.2}$ events. The cross section measured is ${ }^{8}$ :

$$
\sigma(p \bar{p} \rightarrow W W)=14.6_{-5.1}^{+5.8}(\text { stat })_{-3.0}^{+1.8}(\text { syst }) \pm 0.9(\text { lum }) \mathrm{pb}
$$

well in agreement with the predicted cross section:

$$
\sigma(p \bar{p} \rightarrow W W)_{\mathrm{Th} .}=11.3 \pm 1.3 \mathrm{pb}
$$

${ }^{\mathrm{b}} R=\sqrt{\Delta \phi^{2}+\Delta \eta^{2}}$ 

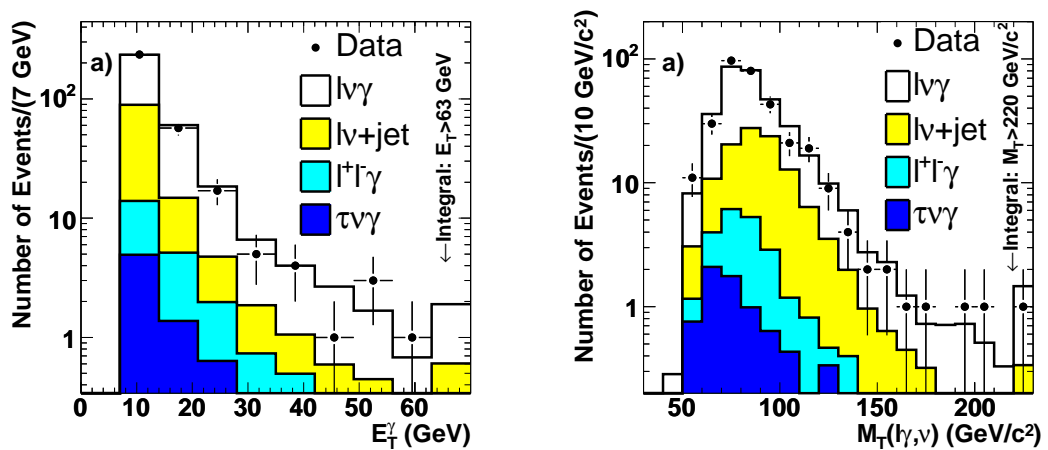

Figure 3. Photon $E_{T}$ (left) and Cluster transverse mass (right) for $W \gamma \rightarrow \ell \nu \gamma$ shown for observed events together with signal and background expectations. Anomalous gauge coupling will increase the high energy tail of the photon $E_{T}$ distribution.

\section{Conclusions}

CDF is producing interesting results in the electroweak sector. Most of them have a precision that is limited by statistics. Therefore we are approaching a period where, with the available datasets, CDF can produce electroweak physics measurements with the smallest single experiment uncertainties. We are also refining the analysis using new methods that will decrease the systematics uncertainties. So far, no SM deviations have been observed.

\section{Acknowledgments}

It is a pleasure to thank all those at CDF and at Fermilab that are working hard for the success of the RunII of the Tevatron. I would also like to thank the organizers of this wonderful Lake Louise Winter Institute.

\section{References}

1. P.J. Sutton et al., Phys Rev D45, 2349 (1992); P.J. Rijken et al., Phys Rev D51 44 (1995); R. Hamberg et al., Nucl. Phys. B359 343, (1991), R.V. Harlander et al., Phys. Rev. Lett. 88201801 (2002).

2. D. Acosta et al. [CDF II Collaboration], Phys. Rev. Lett. 94, 091803 (2005).

3. S. Eidelman et al, Phys. Lett. B 592, 1 (2004).

4. D. Acosta et al. [CDF Collaboration], Phys. Rev. D 71, 051104 (2005).

5. G. Apollinari, et al. Nucl. Instrum. Meth. A412,515-526, (1998).

6. D. Acosta et al. [CDF II Collaboration], Phys. Rev. Lett. 94, 041803 (2005).

7. U. Baur, T. Han and J. Ohnemus, Phys. Rev. D 53 (1996) 1098.

8. D. Acosta et al. [CDF Collaboration], hep-ex/0501050. 